\title{
Impact of Sampling Frequency Offset on PRIME 1.4 PLC Systems Performance
}

\author{
José A. Cortés*, Alfredo Sanz ${ }^{\dagger}$, Luis Díez ${ }^{*}$, Francisco J. Cañete* \\ * Universidad de Málaga, Andalucía Tech, Dpt. Ingeniera Comunicaciones, \\ E.T.S.I. Telecomunicación, Málaga (29071), Spain \\ email: \{jaca, diez, francis\} @ic.uma.es \\ $\dagger$ Microchip Technology Inc., Torre C2, Polígono Puerta Norte, A-23, Zaragoza (50821), Spain \\ email: alfredo.sanz@microchip.com
}

\begin{abstract}
The purpose of this paper is to study the impact of an uncorrected sampling frequency offset (SFO) on the frame error rate (FER) of a PRIME 1.4 system. This is helpful to evaluate the need to implement new algorithms to estimate and correct that SFO or to adopt more simpler strategies that mitigate somehow this problem, like applying in the receiver a shift to the fast-Fourier transform window towards the cyclic prefix part of the symbol.
\end{abstract}

\section{INTRODUCTION}

Power line communications (PLC) is a mature technology for many applications both for broadband and narrowband systems. An interesting compilation of texts devoted to the topic can be consulted in [1]. Smart-grids can be considered the main application of narrowband PLC technology [2] and several standards exist that define multicarrier transmission systems for this purpose, being the most successful ones PRIME (PoweRline Intelligent Metering Evolution) and G3PLC [3], [4] and later IEEE 1901.2 [5], [6] that tries to harmonize both.

One of the novel features of PRIME 1.4 [7] is a bandwidth extension up to $500 \mathrm{kHz}$ organized in eight channels that can be selected independently for the transmission. Other novelty is the support of robust transmission modes with DBPSK and DQPSK that join to the already existent set of differential (in frequency) modulations: DBPSK, DQPSK and D8PSK. These robust modes allow, besides the convolutional coding (with a rate of $1 / 2$ and a constraint length of 7 ), a repetition of the coded symbols 4 times, what implies frames with a longer preamble and header that are called type $B$ frames. As a consequence of this innovation, the common practice of disregarding sampling frequency offset (SFO) correction in commercial PRIME receivers may become an issue and the study of this problem is the main contribution of this work. An additional result is that, although there are several papers that have analyzed the performance of PRIME systems for previous releases [8], [9], to the best author knowledge, it is the first time that FER is evaluated for robust modes of PRIME 1.4 systems under realistic conditions.

The paper structure is as follows. After this introduction, the system model is explained in section II. The effects of an uncompensated SFO are presented in section III and the impact on the system performance is evaluated by estimating the FER in section IV. Finally, some conclusion is given in section $\mathrm{V}$.

\section{SyStem MODEL}

In this work, a model of a PRIME 1.4 system according to [7] is simulated in MATLAB ${ }^{\circledR}$. It contemplates an Orthogonal Frequency Division Multiplexing (OFDM) scheme with a sampling rate of $1 \mathrm{MHz}, 1024$ carriers, 2048-point fast-Fourier transform (FFT) size and a cyclic prefix (CP) of 192 samples. Hence, the OFDM symbol period is $2.24 \mathrm{~ms}$ (2240 samples).

All elements in the transmission chain but the scrambler, which does not have influence in the BER/FER, are included in the model. A maximum likelihood sequence detector, by means of a Viterbi decoder with a finite traceback length of 64 , is implemented in the simulations to perform the convolutional decoding after the differential demodulation. Two possible receivers are considered. The first is an ideal receiver, which provides the log-likelihood ratio (LLR) metric of the demodulated complex symbols to the Viterbi decoder without any quantization (the metric of the soft decision contains one LLR value for each bit in a symbol, i.e. three for D8PSK). The second is a simplified receiver, in which the demodulated bits are estimated from a quantization of the differential angle between consecutive symbols before the decoder. The output of the ideal soft demodulator for the symbols in type B frames, where the repetition code is employed, is obtained by averaging the LLR values corresponding to each repetition. In the simplified receiver the averaging is performed over the quantized values.

In the simulations, it is assumed that the frame is always detected and the preamble is decoded without errors. The BER and FER are calculated, therefore, only for the payload part of the frame. Maximum length frames are considered: 63 payload symbols, for type A frames, and 252 payload symbols, for type B frames.

A simple model, composed of a flat channel plus additive white noise, is employed in the analysis to isolate effects. For a more efficient implementation, the noise is introduced in the frequency domain at the output of the receiver FFT. Hence, the received signal to noise ratio (SNR) is only computed for the used carriers and out-of-band noise is not considered. 


\section{EFFECTS OF AN UNCOMPENSATED SFO}

A sampling frequency offset (SFO) between the oscillators in the transmitter and receiver results in a sampling-time error $\Delta n$ defined as $T_{R x}=(1+\Delta n) T_{T x}$ and measured in unit fractions; where $T_{R x}$ and $T_{T x}$ represent the sampling period at receiver and transmitter, respectively. In PRIME 1.4, and in previous releases as well, the maximum tolerance of the oscillators is $50 \mathrm{ppm}$, what leads to a maximum SFO of 100 ppm.

Let us consider an OFDM system with an FFT size of $N$ points and $X_{k}^{m}$ denotes the complex value (the constellation symbol) associated to the modulation employed in the $k$-th carrier of the $m$-th OFDM symbol (just symbol from now on). Then, after some algebraic manipulations, the complex value obtained at the FFT output in the receiver for such carrier and symbol is given by

$$
\begin{aligned}
& Y_{k}^{m} \approx X_{k}^{m} \cdot \frac{1}{N} \frac{\sin (\pi k \Delta n)}{\sin \left(\frac{\pi}{N} k \Delta n\right)} \cdot \\
& \cdot e^{j \frac{2 \pi}{N}\left[k \Delta n \cdot d_{m}+k \Delta n\left(\frac{N-1}{2}\right)\right]}+ \\
&+\sum_{\substack{p=-(N / 2-1) \\
p \neq k}}^{N / 2} X_{p}^{m} \frac{1}{N} \frac{1-e^{j[2 \pi p \Delta n+2 \pi(p-k)]}}{1-e^{j\left[\frac{2 \pi}{N} p \Delta n+\frac{2 \pi}{N}(p-k)\right]}} \\
& \cdot e^{j \frac{2 \pi}{N} p \Delta n \cdot d_{m}}
\end{aligned}
$$

where $d_{m}$ is the index of the first sample of the $m$-th symbol w.r.t. the frame beginning.

As observed in the first term in (1), the FFT output exhibits an amplitude reduction (that corresponds to the factor $\sin () / \sin ())$ and also a phase rotation with two components. The first one, $\Delta \phi_{k}^{m}=\frac{2 \pi}{N} k \Delta n \cdot d_{m}$, is due to the samplingtime error accumulated from the frame beginning to the FFT window beginning. This one increases with the carrier index and the symbol index. The second one, $\Phi_{k}=\frac{2 \pi}{N} k \Delta n\left(\frac{N-1}{2}\right)$, is caused by the error accumulated along the samples used in the FFT calculation and, hence, it only depends on the carrier index. The second term in (1) is the sum of an additional degradation due to the contribution of the complex values transmitted in the remaining carriers that will be referred to as intrasymbol intercarrier interference (ICI).

The expression in (1) is valid under the condition that the time shift of the symbols caused by the SFO is small enough to consider that the FFT window contains only samples of the desired symbol. However, without an adequate compensation, the symbols will be delayed or advanced w.r.t. the FFT window and finally the FFT will be calculated over samples of two adjacent symbols. This fact will result in additional ICI, which is denoted as intersymbol ICI (not contemplated in (1)) to remark its different nature from the intrasymbol ICI.

\section{A. Constellation rotation}

The value of $\Phi_{k}$ that appears in (1) is much smaller than the one of $\Delta \phi_{k}^{m}$ and hence, the phase error experienced by the

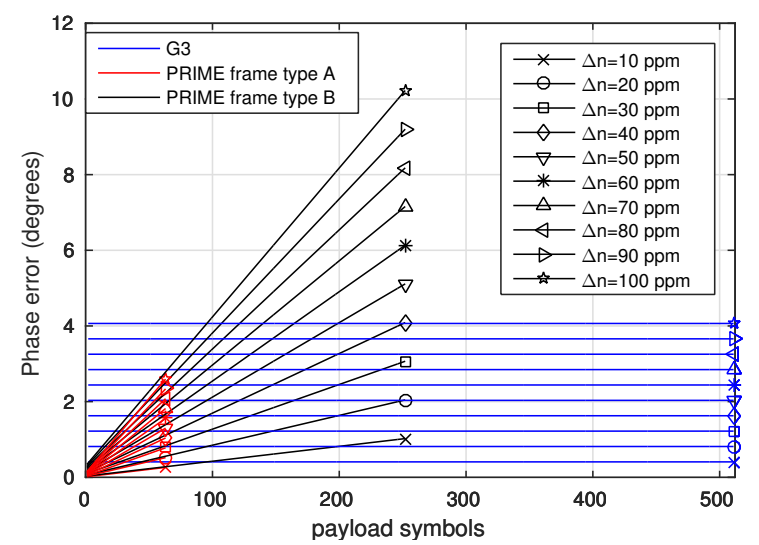

Figure 1: Phase error after differential demodulation.

received complex symbols in the carrier $k$ can be approximated by

$$
\Delta \phi_{k}^{m}=\frac{2 \pi}{N} k \Delta n\left[d_{0}+(N+c p) m\right]
$$

where $c p$ denotes the cyclic prefix length measured in samples and $d_{0}$ the index of the payload first sample w.r.t. the starting point of the frame. The transmission is consider to start at symbol $m=0$.

In PRIME, a differential modulation in the frequency domain is employed and therefore, at the output of the differential demodulation algorithm, the phase error is

$$
\Delta \phi_{k}^{m}-\Delta \phi_{k-1}^{m}=\frac{2 \pi}{N} \Delta n\left[d_{0}+(N+c p) m\right] .
$$

It is worth mentioning the difference between PRIME and G3 compliant receivers in this aspect. In the latter case, it is used a classical differential modulation in time, which leads to a phase error of

$$
\Delta \phi_{k}^{m}-\Delta \phi_{k}^{m-1}=\frac{2 \pi}{N} \Delta n(N+c p) k .
$$

As observed, in PRIME the phase error is independent of the carrier index. All the carriers in a given symbol experience the same error. Hence, there is no difference between the carriers in channel 8 and in channel 1. However, the longer the symbol, the larger the phase error. Conversely, in G3, all symbols exhibit the same phase error, but the higher the carrier index the larger the phase error. These differences between both standards can be observed in Fig. 1, where the phase error after the differential demodulation (between two consecutive carriers) is depicted vs the index of the symbol in the payload, for different sampling-time errors.

It must be noted that in G3 the maximum allowed SFO is 50 ppm (half than in PRIME) and the phase rotation is bounded to two degrees approximately. In PRIME, the maximum phase error is obtained for type B frames, because they are the longest ones, and it is bounded to ten degrees approximately. This type of frames are only used with the more robust modulations DBPDK and DQPSK. For this latter modulation, half the angle of the decision region is 45 degrees and so a 


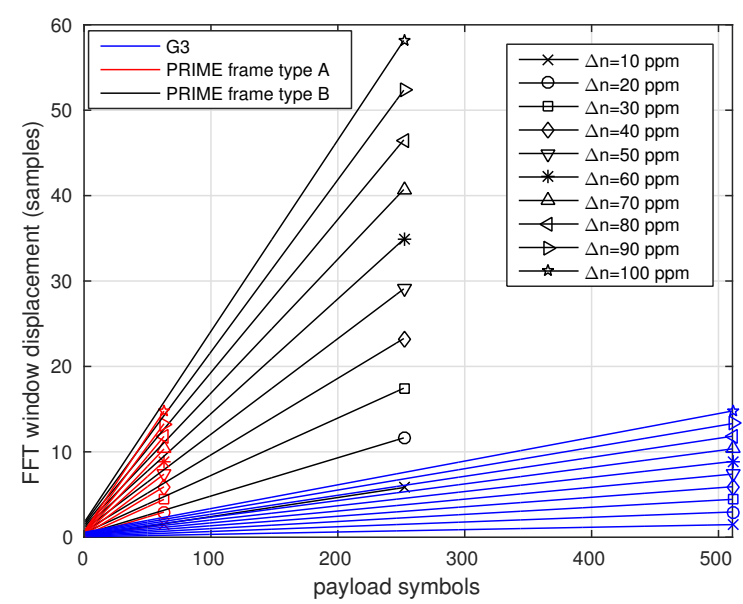

Figure 2: Displacement of the FFT window.

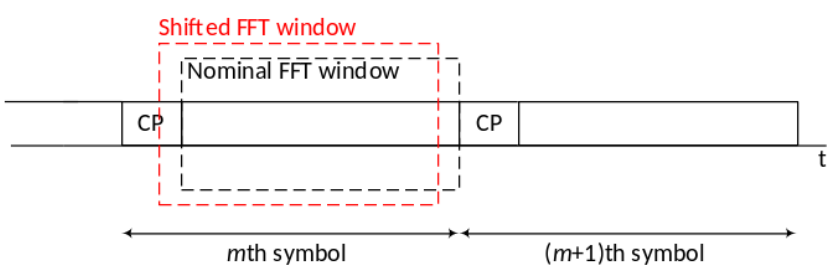

Figure 3: Diagram of the proposed FFT window shift at receiver.

phase error of 10 degrees represent a $22 \%$ of displacement in the region, although this error is only reached in the last symbols of the frame. It can be concluded that the influence of the rotation due to an uncompensated SFO may be modest in most modulations except for DQPSK in which a further study is needed.

\section{B. Symbols drift with respect to FFT window}

As mentioned, the shift of the OFDM symbols w.r.t. the FFT window due to sampling-time error is the cause of intersymbol ICI and this effect in analyzed in Fig. 2 for the two PLC standards. In PRIME 1.4 compliant systems, that shift can be of 60 samples for the robust modes of type B frames and of 15 samples in the normal ones. This latter value is short enough to be mitigated by the CP, provided that the FFT window at the receiver is shifted towards the $\mathrm{CP}$ these same 15 samples, as shown in Fig. 3. By doing so, in case of an uncompensated SFO two possible situations appear. If the symbols move right, still no samples of the next symbol will enter the FFT window. On the contrary, if the symbols move left, 30 samples of the CP will be taken in the FFT window and then, only 162 samples will remain to absorb the channel impulse response effect (and to avoid intersymbol interference -ISI- and intersymbol ICI).

In the robust modes, though, the FFT must be shifted 60 samples towards the $\mathrm{CP}$ and then, in case the symbols move left 60 samples (due to sampling-time error) the effective $\mathrm{CP}$ that remains is of 72 samples. This is yet longer than the

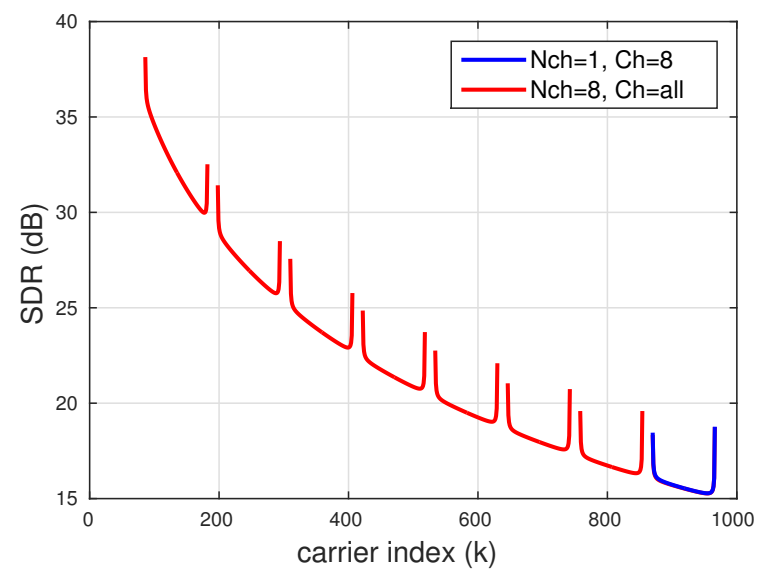

Figure 4: SDR due to intrasymbol ICI vs carrier frequency index. Curves are presented for the case that all eight channels are used or when just the 8th channel is used, whose curve superimposes the previous one.

nominal CP in G3 systems, but may reduce the PRIME system performance respect to the case with no compensation for the SFO. Therefore, a trade-off appears between the level of ISI and ICI, hence it would be convenient to evaluate the system degradation with a shorter than 60 samples FFT shift.

\section{Intrasymbol ICI}

The SFO cause an expansion/compression of the signal spectrum, being $\Omega$ the new index of discrete-time frequency and $\Omega^{o}$ the nominal one, both are related by means of $\Omega=\Omega^{o} \frac{f_{s}}{f_{o}^{o}}=\Omega^{o}\left(1+\Delta f_{s}\right)$, with $f_{s}=f_{s}^{o}\left(1+\Delta f_{s}\right)$, where $f_{s}$ and $f_{s}^{o}$ represent the actual sampling frequency and the nominal one, respectively. This expansion/compression of the spectrum breaks the orthogonality among carriers, what results in ICI. The signal to distortion ratio (SDR) is used to evaluate the impact of intrasymbol ICI and is defined as the ratio between desired signal and ICI power levels at each carrier (this latter is calculated from (1))

$$
\frac{S D R_{k}=}{\left.\left.\sum_{m}\left|\sum_{\substack{p=-(N / 2-1) \\ p \neq k}}\right| X_{k}^{m}\right|^{2} \frac{1}{N} \frac{1-e^{j[2 \pi p \Delta n+2 \pi(p-k)]}}{1-e^{j\left[\frac{2 \pi}{N} p \Delta n+\frac{2 \pi}{N}(p-k)\right]}} e^{j \frac{2 \pi}{N} p \Delta n \cdot d_{m}}\right|^{2}}
$$

In Fig. 4, the obtained SDR values for a SFO of $100 \mathrm{ppm}$ are plotted. A decaying behavior with frequency is observed: the higher carriers experience more ICI because they are more sensitive to the expansion/compression of the frequency axis. In addition, at the edge of the channels the SDR is better because there are no interfering adjacent carriers at one side, where a guard band is established. The presence of guard bands and the fact that ICI is mainly due to the adjacent carriers, make both curves in Fig. 4 indistinguishable: there 


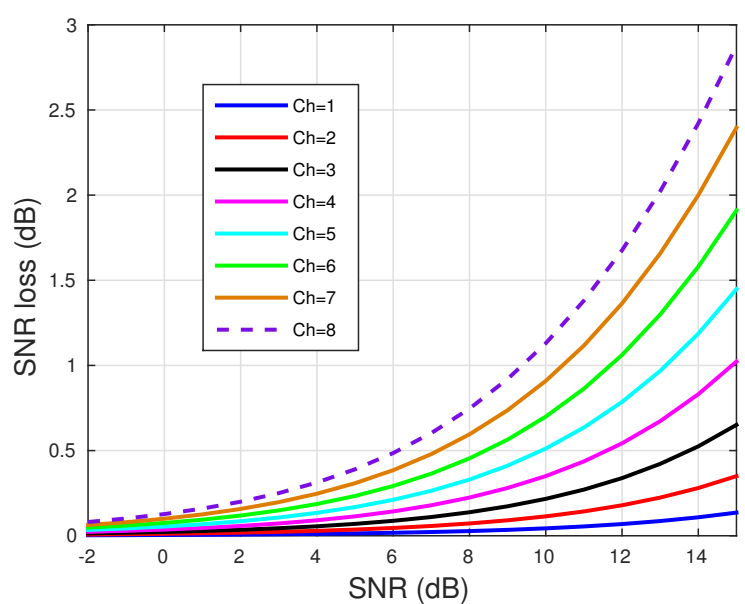

Figure 5: Equivalent SNR loss due to intrasymbol ICI addition for each of the channel bands.

is no practical difference in using just the higher channel or all channels simultaneously.

The ICI impact on the system performance can be neglected if the SDR level is about $10 \mathrm{~dB}$ over the SNR level (because the ICI will be masked by the noise floor). To assess that, in Fig. 5 the equivalent SNR loss in the system when including the intrasymbol ICI as an additional noise term is plotted. For each channel, the worst case is considered, which corresponds to the carrier with lower SDR. As observed, in the higher channels the loss is very important and larger modulations like D8PSK will exhibit a notable performance reduction. However, in robust modulations the ICI has little influence because they work at low SNR levels (around $4 \mathrm{~dB}$ or even less).

\section{SYSTEM PERFORMANCE ANALYSIS}

In this section, a more detailed analysis is presented by evaluating the system performance degradation when the SFO is not corrected. To this end, the increase of the FER is calculated by means of simulations. First, the impact of the phase rotation and intersymbol ICI on the performance is analyzed (they can not be separated in the simulations), and the proposal of shifting the FFT window to alleviate it is discussed as well. Second, the effect of the intrasymbol ICI is assessed, which can not be mitigated unless a correction of the sampling time is performed.

The effect of the SFO is introduced in the simulations, on the one hand, in the time domain, by shifting the symbols w.r.t. the FFT window of the receiver; and on the other hand, in the frequency domain, by applying a phase rotation to the complex received values. The first one models the integer part of the displacement caused by the SFO and the second one the fractional part. The advantage of this strategy is to avoid the computational cost of performing a fractional rate interpolation in the time domain. However, two disadvantages appear: it is not considered neither the intrasymbol ICI, due to the calculation of the FFT over samples that are not evenly

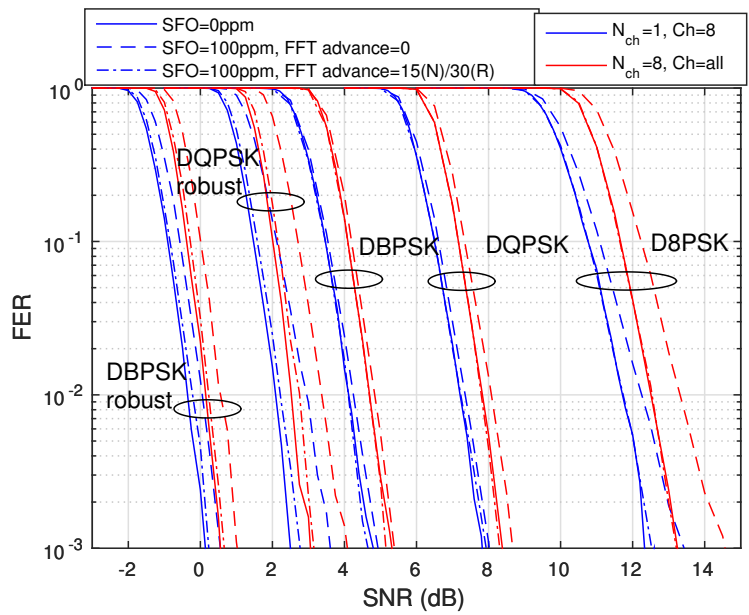

Figure 6: FER estimated for an ideal receiver under several conditions.

spaced in continuous time, nor the attenuation of the complex values. The latter is not significant (due to the magnitude of the factor $\sin () / \sin ()$ in (1)), because for an SFO of $100 \mathrm{ppm}$ the loss is below $0.15 \mathrm{~dB}$ for all carriers. Nevertheless, the effect of the intrasymbol ICI suffered by each carrier has been modeled by means of a Gaussian white random variable, additive to the noise, and whose variance is given by the corresponding ICI power. The Gaussian assumption is reasonable taking into account the high number of carriers even when only a channel is used.

\section{A. Influence of the constellation rotation and intersymbol ICI}

The study has been carried out for both receivers, ideal and simplified, under three conditions: a) without SFO (as a reference), b) with a SFO of $100 \mathrm{ppm}$, and c) with a SFO of 100 ppm but shifting the FFT window at receiver to compensate for that. In the third condition, the FFT advance towards the $\mathrm{CP}$ is set to two different values regarding the mode employed: 15 samples for normal modes (type A frames) and 30 samples for robust modes (type B frames). In the former case, the advance compensates for the whole shift that OFDM symbols may exhibit due to SFO and there is no intersymbol ICI. In the latter case, the advance only supports half the maximum eventual shift of the symbols (as seen in Fig. 3). In that case, there will be also ISI besides intersymbol ICI because some samples of the next symbol are included in the FFT.

1) Ideal receiver: In Fig. 6, the estimated FER for a system with an ideal receiver under two different situations is shown: when only one channel is transmitted (the highest one), denoted as $(\mathrm{Nch}=1, \mathrm{Ch}=8)$ or when all channels are transmitted, denoted as $(\mathrm{Nch}=8, \mathrm{Ch}=\mathrm{all})$. The worse behavior of the FER when all channels are used needs an explanation. Actually, the BER is the same for both situations, but since all simulations have been carried out with frames of the maximum length (in symbols), the number of bits in a frame when all channels are used is 8 times larger than when only one channel 


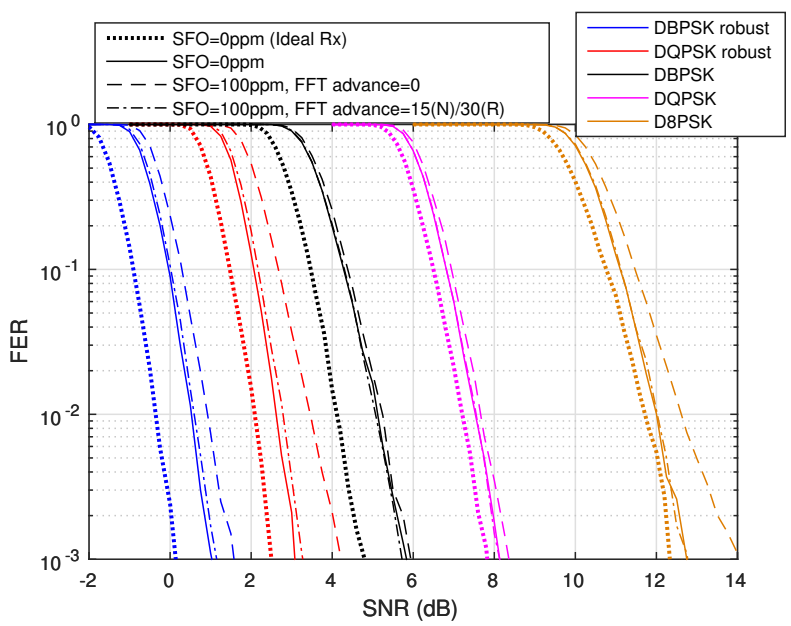

Figure 7: FER estimated for a simplified receiver under several conditions.

\begin{tabular}{|c|c|}
\hline modulation & SNR loss $(\mathrm{dB})$ \\
\hline robust DBPSK & 0.88 \\
robust DQPSK & 0.5 \\
DBPSK & 1 \\
DQPSK & 0.3 \\
D8PSK & 0.3 \\
\hline
\end{tabular}

Table I: SNR loss of the simplified vs the ideal receiver, considering a $\mathrm{FER}=1 \%$ and $\mathrm{SFO}=0 \mathrm{ppm}$.

is used and hence the probability of frame error is also higher. Under condition (b), i.e. SFO occurs and the FFT window is not shifted at receiver, the performance degradation in all modulations is bounded to $1 \mathrm{~dB}$ if a $\mathrm{FER}=1 \%$ is assumed. Under condition (c), when SFO influence is mitigated by shifting the FFT window, the degradation is bounded to $0.1 \mathrm{~dB}$ in all modulations for a FER $=1 \%$. For normal modes, as there is no intersymbol ICI and the obtained FER curve is almost superimposed to the one of condition (a) without SFO, it can be concluded that the constellation rotation has no practical effect.

2) Simplified receiver: In Fig. 7, the estimated FER for a system with a simplified receiver is presented. The simulated conditions are the same as above although, the FER curve for the ideal receiver without SFO is also included as a reference. In all modulations, the needed SNR increment to guarantee a $\mathrm{FER}=1 \%$ under condition (b) is also approximately bounded to $1 \mathrm{~dB}$, as for the ideal receiver; and under condition (c) is again negligible. Hence, the proposal of shifting the FFT window solves the problem.

In order to compare their performance, in Table I the SNR loss between the simplified receiver and the ideal one is summarized for all modulations. It is observed that the worse case corresponds to DBPSK, both in robust and normal modes, and the loss is close to $1 \mathrm{~dB}$.

\section{B. Overall performance}

To evaluate the degradation in the system performance also due to intrasymbol ICI a semi-analytic procedure has been

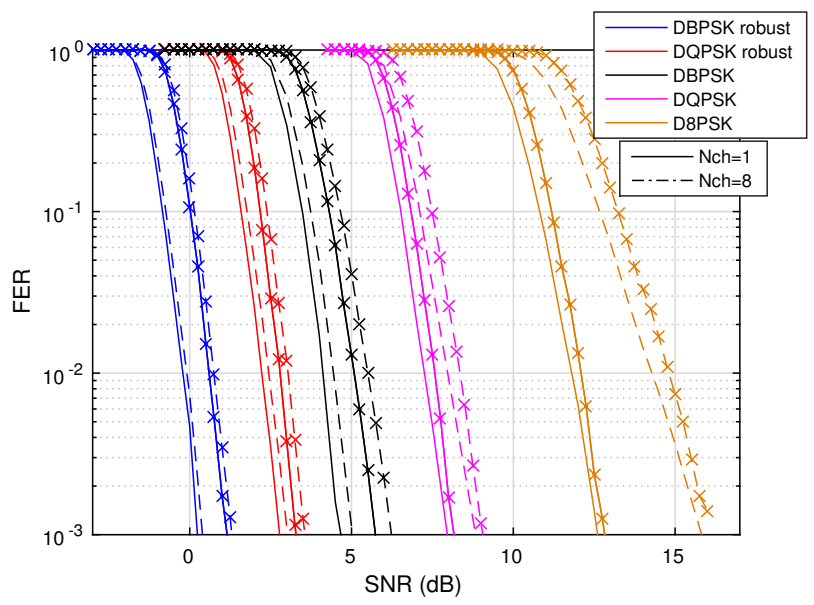

Figure 8: FER estimated for the ideal and simplified receiver. Lines with markers corresponds to the simplified receiver and lines without markers to the ideal receiver. Curves for the 8th channel and the 1st channel are included.

followed, by modeling the ICI as a Gaussian random variable additive to the noise. To this end, the signal to noise and distortion ratio (SNDR) at each channel is computed as

$$
\begin{aligned}
S N D R^{i} & =\frac{S}{N+I C I_{\max }^{i}} \\
& =\left(\frac{1}{S N R}+\frac{1}{S N R_{\min }^{i}}\right)^{-1},
\end{aligned}
$$

where $S$ and $N$ are the power level of the desired signal and the noise respectively, and $I C I_{\max }^{i}$ corresponds to the power level of the ICI for the worst carrier of the $i$-th channel that is calculated for each of the carriers according to (1). Then, the FER of the $i$-th channel for each of the modulation schemes is calculated as

$$
F E R^{i}(S N R)=F E R\left(S N D R^{i}\right),
$$

where the expression $F E R(x)$ represents the function obtained by simulation in subsection IV.A for a $\mathrm{SNR}=x$.

It is worth mentioning that, despite this is a quite efficient method to evaluate this degradation, it may underestimate the system performance since the ICI in all carriers of a channel is taken as the one in the worst carrier ${ }^{1}$.

The FER calculated in this way, when the intrasymbol ICI is added to a system that incorporates the proposal of shifting the FFT window 15 samples towards the $\mathrm{CP}$ for normal modes and 30 samples for robust modes, is presented in Fig. 8. Curves are plotted for channels 1 and 8 , for the case of both receivers and for all transmission modes. It can be observed that differences between the lower and higher channels (solid and dashed lines respectively), for both receivers, are not significant in low order modulations, because the effect of SFO is not so

\footnotetext{
${ }^{1}$ However, although the BER will be worse than the actual one, the overestimation of the FER maybe modest, because a frame error occurs both when there is only one bit error in a frame and when there are lots of them.
} 


\begin{tabular}{|c|c|c|}
\hline modulation & ideal Rx & simplified Rx \\
\hline robust DBPSK & 0.1 & 0.15 \\
robust DQPSK & 0.22 & 0.25 \\
DBPSK & 0.34 & 0.4 \\
DQPSK & 0.75 & 0.8 \\
D8PSK & 2.48 & 2.7 \\
\hline
\end{tabular}

Table II: SNR loss $(\mathrm{dB})$ between the 1st and 8th channels in the ideal and simplified receiver, considering a $\mathrm{FER}=1 \%$ and $\mathrm{SFO}=100 \mathrm{ppm}$.

important. However, the differences are remarkable in D8PSK, which is quite more sensitive to the uncompensated SFO.

In table II, this analysis is summarized, by measuring the SNR loss between the higher and lower channels for a FER $=1 \%$ and a SFO of $100 \mathrm{ppm}$. The degradation is negligible for the robust modes and DBPSK in normal mode. However, the uncompensated SFO is considerable in normal mode DQPSK and can be very important in D8PSK, with a $2.5 \mathrm{~dB}$ loss for the ideal receiver and a $2.7 \mathrm{~dB}$ loss for the simplified receiver.

A trade-off appears between receiver simplicity and transmission data rates because, the use of larger constellations may require the implementation of time-domain algorithms to correct the SFO. The ICI becomes relevant, especially when the communication is established over high SNR channels and, in these cases, in PRIME it is allowed to transmit even without coding to maximize the data rate. Under such conditions the performance of D8PSK may be compromised without a correction of the SFO.

\section{CONCLUSION}

In this paper, the performance of an OFDM system according to PRIME 1.4 is analyzed in presence of an uncompensated SFO. The different effects caused by the SFO are studied separately: received constellation attenuation, phase rotation, intrasymbol ICI due to loss of orthogonality among carriers and intersymbol ICI and ISI due to misalignment of the FFT window and received symbols. The obtained results indicate that SFO effects can be mitigated almost completely in the two lower channels by shifting the FFT window some samples towards the $\mathrm{CP}$. The magnitude of the proposed shift changes for type A or type B frames.

However, the system performance degradation increases with the channel index due to the intrasymbol ICI, which can only be dealt with SFO compensation algorithms in the timedomain. The performance remains essentially unaltered in the robust modes, where the channel noise dominates over the intrasymbol ICI, but for D8PSK the FER of channel 8 degrades by $2.7 \mathrm{~dB}$. Hence, under certain circumstances, systems can exhibit poorer performance in PRIME 1.4 than in PRIME 1.3 unless the receiver design improves.

\section{ACKNOWLEDGMENT}

The authors thank Microchip Technology Inc. for the support provided to accomplish this work.

\section{REFERENCES}

[1] IEEE Communications Society, "Best Reading on Power Line Communications". Available online: http://www.comsoc.org/ bestreadings/topic/powerline-communications

[2] S. Galli, A. Scaglione, Z. Wang "For the grid and through the grid: The role of power line communications in the smart grid." Procedings of the IEEE, Special Issue on Smart Grid, vol. 99, no. 6, pp. 998?1027, June 2011.

[3] A. Sendin, I. Peña and P. Angueira, "Strategies for Power Line Communications Smart Metering Network Deployment," Energies 2014, 7, 2377-2420.

[4] ERDF G3-PLC "PLC G3 Physical Layer Specification". Available online: http://www.g3-plc.com

[5] IEEE P1901.2-2013, "IEEE standard for low-frequency (less than 500 $\mathrm{kHz}$ ) narrowband power line communications for Smart Grid applications," IEEE Standards Association, 2013.

[6] J. LeClare, A. Niktash, V. Levi, "An Overview, History, and Formation of IEEE P1901.2 for Narrow-band OFDM PLC", Maxim AN 5676. Available online: https://www.maximintegrated.com/ en/appnotes/index.mvp/id/5676

[7] PRIME Specification Revision 1.4, Specification for PoweRline Intelligent Metering Evolution, 2014. Available online: http://www.primealliance.org

[8] I. Berganza, A. Sendin, A. Arzuaga, M. Sharma, B. Varadarajan, "PRIME on-field deployement," in Proceedings of the IEEE International Conference on Smart Grid Communications, Brussels (Belgium) 2011, p. 297302.

[9] M. Wolkerstorfer, B. Schweighofer, H. Wegleiter, D. Statovci, H. Schwaiger and W. Lackner, "Measurement and simulation framework for throughput evaluation of narrowband power line communication links in low-voltage grids," Elsevier Journal of Network and Computer Applications, 59 (2016), pp. 285-300. 